\title{
Chemical composition of semi-regular variable giants. II. ${ }^{\star}$ (Research Note)
}

\author{
N. E. Britavskiy ${ }^{1}$, S. M. Andrievsky ${ }^{1,2}$, S. A. Korotin ${ }^{1}$, and P. Martin ${ }^{3}$ \\ 1 Department of Astronomy and Astronomical Observatory, Odessa National University, T.G. Shevchenko Park, 65014 Odessa, \\ Ukraine, and Isaac Newton Institute of Chile, Odessa Branch, Ukraine \\ e-mail: britvavskiy@gmail.com; scan@deneb1.odessa.ua; serkor@skyline.od.ua \\ 2 GEPI, Observatoire de Paris-Meudon, 92125 Meudon Cedex, France \\ 3 WIYN Observatory, 950 N. Cherry Ave. Tucson, AZ 85719, USA \\ e-mail: pmartin@wiyn.org
}

Received 30 April 2010 / Accepted 5 July 2010

ABSTRACT

\begin{abstract}
Aims. The aim of this work was to derive the elemental abundances in four stars classified as semiregular variables of the type "d" (SRd). These stars should presumably belong to the Galactic halo population.

Methods. Elemental abundances have been derived from both local thermodynamical equilibrium (LTE) and non-local thermodynamical equilibrium (NLTE) analyses, applied to high-resolution ( $R \cong 80000)$ spectra obtained with the CFHT ESPaDOnS spectrograph. Results. We have derived the abundances of 29 chemical elements in V463 Her, V894 Her, CW CVn, and MS Hya, and arrived at the following conclusions.

Conclusions. The stars of our programme have a chemical composition that does not agree with their presumable status as metaldeficient halo giants. All studied SRd giants have relative-to-solar elemental abundances typical of the thick/thin Galactic disc stars.
\end{abstract}

Key words. stars: variables: general - stars: abundances

\section{Introduction}

According to the General Catalogue of Variable Stars (GCVS) more than two hundred giants of the F, G, and K-M spectral classes show a semi-regular (i.e. quasi-regular) light variation with amplitudes in the range of 0.1 to 4 mag, and "periods" spanning from 30 to 1100 days. These giant stars are called semiregular variables of type "d" (SRd). There are no very precise classification criteria for SRd stars, but nevertheless there are some photometric characteristics that allows one to distinguish SRd stars from other representatives of the general class of semiregular variables ( $\mathrm{SRa}, \mathrm{SRb}$, and $\mathrm{SRc}$ stars).

The kinematics of some of these stars support their halo origin. A few results on abundance analyses of SRd stars available in the literature (see the introduction in Andrievsky et al. 2007a) also support this statement. The range of observed characteristics among the stars of this type (periods and magnitude variation) is rather broad and may be partially influenced by metallicity. For instance, phenomenologically classical cepheids resemble W Vir type stars, or RR Lyr type stars (in the past all of them had the common name "cepheids"), although the stars of these types actually belong to different stellar populations (thin disc, thick disc, halo). The differences in their metallicity explains partly the differences in their pulsational characteristic. The same is valid also for $\delta$ Sct and SX Phe type stars.

* Based on observations obtained at the Canada-France-Hawaii Telescope (CFHT), which is operated by the National Research Council of Canada, the Institut National des Sciences de l'Univers of the Centre National de la Recherche Scientifique of France, and the University of Hawaii.
Because the SRd phenomenon was traditionally considered to be connected with the stellar population of the halo, stars of that type should have metallicities well below solar. Crimi \& Mantegazza (1984) investigated the SRd giant IS Gem and arrived at the conclusion that the SRd class is not homogeneous. There should exist a possible subdivision of this class between two different types of objects, one of Population I and the other of Population II. Because of the ill-defined classification criteria, this class comprises a mixture of members of the different evolutionary stages and masses (Walhgren 2003).

How chemically homogeneous is the class of SRd-type stars? To answer this question we need to significantly enlarge the number of spectroscopically studied stars of this type. Up to the present only a few papers on this topic have been published (Andrievsky et al. 1985; Giridhar et al. 1998, 1999, 2000; Andrievsky et al. 2007a). In this paper we present the results of a detailed study of four stars of SRd type.

\section{Observations}

We selected several SRd stars listed in GCVS for our observations using the Canada-France-Hawaii 3.6-m telescope. Observations were carried out during three nights in 2005 with the fiber-fed ESPaDOnS spectrograph which is equipped with a EEV $2000 \times 4500 \mathrm{CCD}$ camera (binned $1 \times 1$ ). The resolving power provided by this combination is about 80000 and the spectral range extends from $3700 \AA$ to $10500 \AA$. The list of the stars and some of their characteristics are given in Table 1. For all stars studied the $S / N$ ratio within the observed spectral fragments is higher than 100 . The spectra, first processed through the CFHT ESPaDOnS reduction pipeline, were extracted with 

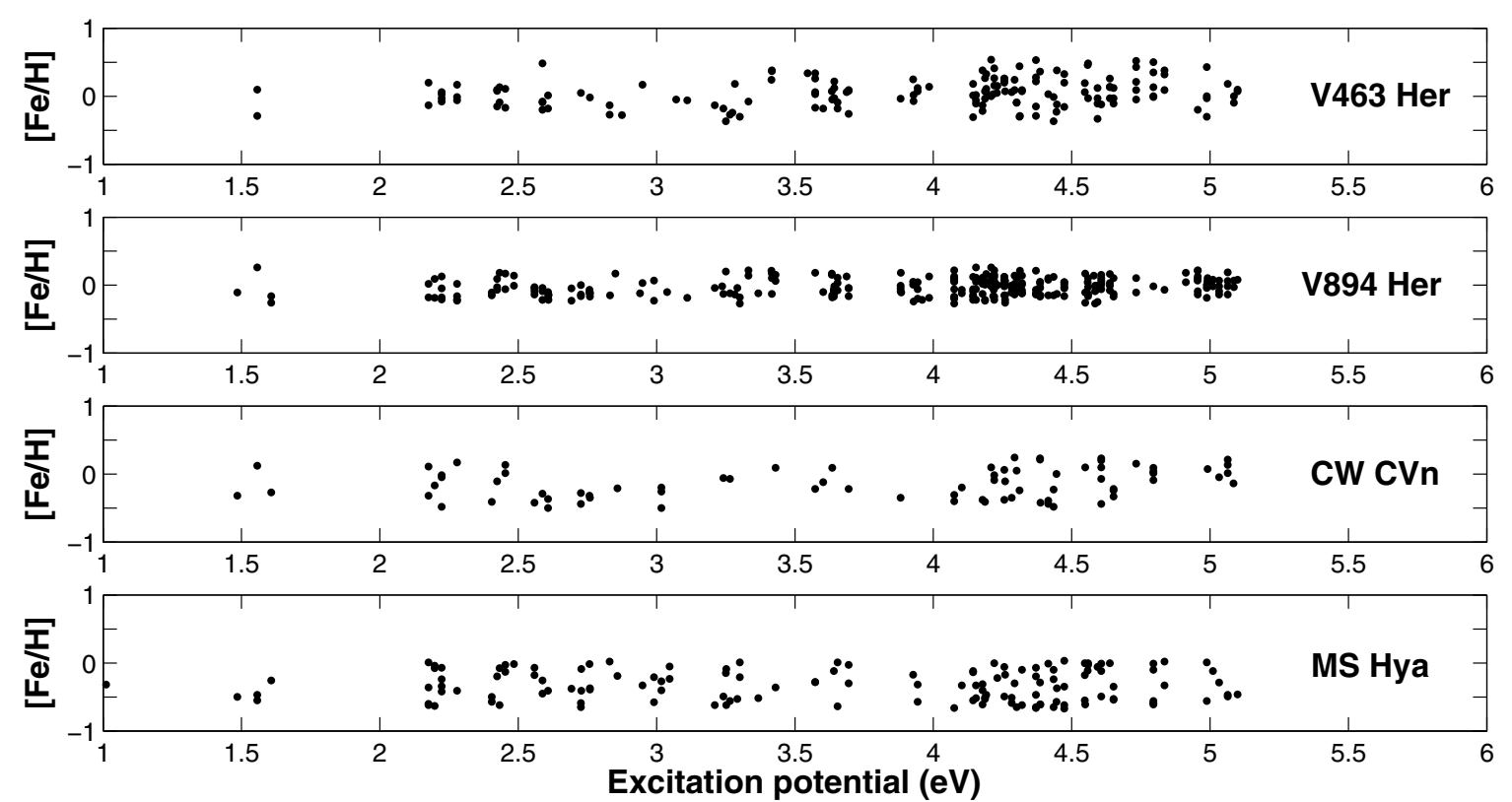

Fig. 1. Relative-to-solar iron abundance from Fe I lines versus excitation potential for the stars of our programme. As is the convention in spectroscopic literature, hereafter we denote the relative-to-solar abundance of a certain element as $[\mathrm{El} / \mathrm{H}]=\log \epsilon(\mathrm{El})-\log \epsilon(\mathrm{El}) \odot$

Table 1. Characteristics of programme stars: visual magnitudes, effective temperatures, surface gravities (logarithm) and microturbulent velocities.

\begin{tabular}{cccccc}
\hline \hline Star & $V$ & $T_{\text {eff }}, \mathrm{K}$ & $\log g$ & $V_{\mathrm{t}}, \mathrm{km} \mathrm{s}^{-1}$ & $\mathrm{Rem}$ \\
\hline V463 Her & $8.46-?$ & 4845 & 2.20 & 1.20 & SRd \\
V894 Her & $8.17-8.32$ & 4620 & 2.00 & 1.10 & SRd \\
CW CVn & $7.00-7.24$ & 3950 & 0.00 & 1.90 & SRd \\
MS Hya & $6.99-7.09$ & 3900 & 0.00 & 1.10 & SRd \\
\hline
\end{tabular}

Notes. The classification of the stars is from GCVS.

standard IRAF procedures. Additional work with the spectra (continuum placement, equivalent width measurements, etc.) was performed with the DECH20 code (Galazutdinov 1992).

\section{Analysis}

The equivalent widths of the spectral lines selected for our analysis were measured with a Gaussian approximation. For all stars in our sample, the absorption lines look narrow and symmetric, and this enabled us to measure the line equivalent widths with a high accuracy.

The list of the unblended or weakly blended lines has been selected from the VALD database (http: //ams.astro. univie.ac.at/vald/). For all those lines, this database lists the excitation potentials, oscillator strengths, and broadening parameters. Our spectroscopic analysis was based on LTE Kurucz's atmosphere models (Kurucz 1992). For all investigated chemical elements, except for carbon, oxygen, sodium, magnesium, aluminum, sulfur, potassium, and barium, the elemental abundances were derived with the help of Kurucz's LTE WIDTH9 code. Abundances of the elements listed above were found using the NLTE spectrum synthesis based on the modified version of the MULTI code (see Carlsson 1986 for the MULTI description; and Korotin et al. 1999a,b; Korotin \& Mishenina 1999; Andrievsky et al. 2001, 2007b, 2008, 2009, 2010, and references therein, for the description of the code modification and applied atomic models).

The effective temperatures $T_{\text {eff }}$ for each programme star were estimated using the line-depth-ratios. The line-depth-ratios method, based on sensitive temperature indicators, was developed for giant stars by Kovtyukh et al. (2006). The method is independent of the interstellar reddening, and only marginally dependent on individual characteristics of the stars, such as rotation, microturbulence, and metallicity. The mean error in $T_{\text {eff }}$ determination is not larger than $100-150 \mathrm{~K}$. To verify the results of the temperature determination, we have applied the standard procedure of avoiding any dependence between the iron abundance from Fe I lines and their excitation potentials (Fig. 1). Results are presented in Table 1.

As it was shown by Kovtyukh \& Andrievsky (1999), the best and most reliable way to estimate the microturbulent velocity in the atmosphere of supergiant/giant stars (and thus to subsequently reliably determine the gravity value) is to use the Fe II lines. Unfortunately, there are only a few Fe II lines available in the spectra of one cool star (for another cool star only a few lines of Ti II can be used), while the number of Fe II lines in hotter programme stars is also not very high. Therefore we applied the standard method of the $V_{\mathrm{t}}$ determination based on the use of Fe I lines. Results are shown in Fig. 2. The stellar atmosphere parameters of our objects are given in Table 1. Because we used the VALD's compilation of oscillator strengths, we have eliminated possible random errors in the derived abundances by comparing the stellar elemental abundances with the solar values, which have been determined with the same log $g f$ values, the solar atmosphere model from the Kurucz's grid, and a microturbulent velocity of $1.3 \mathrm{~km} \mathrm{~s}^{-1}$. The abundance results are presented in the next section.

To verify the accuracy of the abundances derived from our equivalent width analysis, we also applied a synthetic spectrum technique (Tsymbal 1996, LTE code) in order to take into account the possible blending of some lines and continuum level distortion caused by molecular bands in some cases. This check 

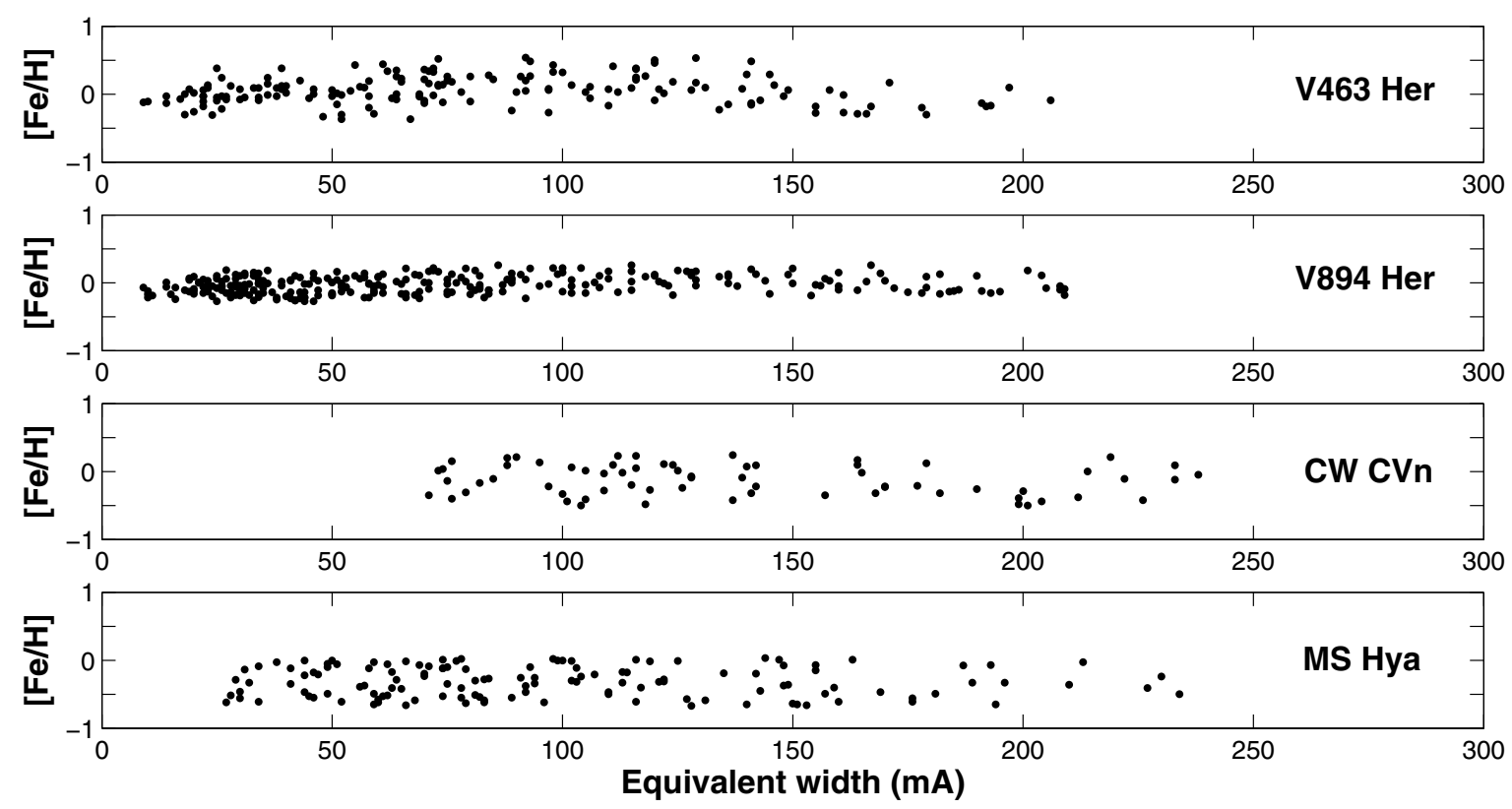

Fig. 2. Relative-to-solar iron abundance from the Fe I lines versus the equivalent width for the four stars of our programme.
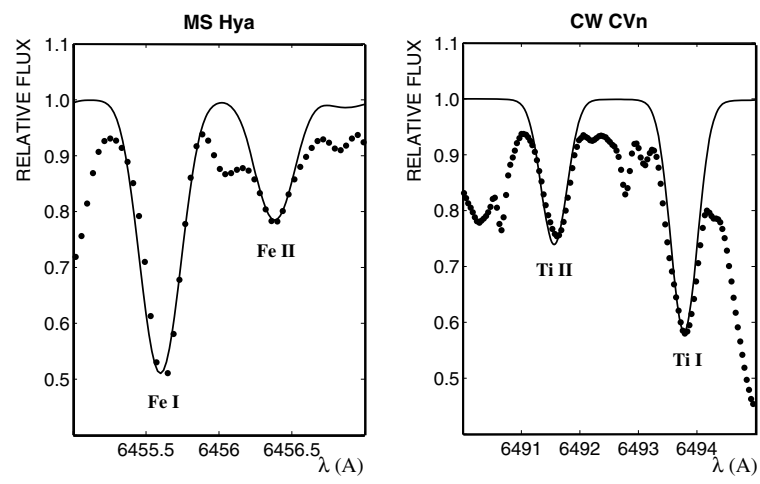

Fig. 3. LTE Fe I/Fe II and Ti I/Ti II line profiles fitted to observed spectrum. Relative flux $=F_{\lambda} / F_{\mathrm{c}}$.

was absolutely necessary for two cooler stars of our sample, whose spectra show a presence of many molecular features. In addition, only a few Ti II or Fe II lines can be found in their spectra, but these lines are important for the correct determination of the gravity. Therefore those lines were carefully analysed with the WIDTH and SYNTH codes. In Fig. 3 we show the calculated synthetic spectrum for two cooler stars with examples for the $\mathrm{Fe} \mathrm{I} / \mathrm{Fe}$ II and $\mathrm{Ti} \mathrm{I} / \mathrm{Ti}$ II lines.

The NLTE abundances were found by fitting the NLTE line profiles generated with a modified version of the MULTI code to observed line profiles. When it was necessary the NLTE code was combined with the LTE synthetic spectrum in order to take into account the blending of the lines treated in NLTE with lines of other species. Some examples of NLTE profiles fitted to observed spectra are given in Fig. 4.

\section{Abundance results}

Tables 2 and 3 list the relative-to-solar abundances for the stars of our sample. Graphically, the distribution of the chemical elements in the studied stars is shown in Figs. 5 and 6.
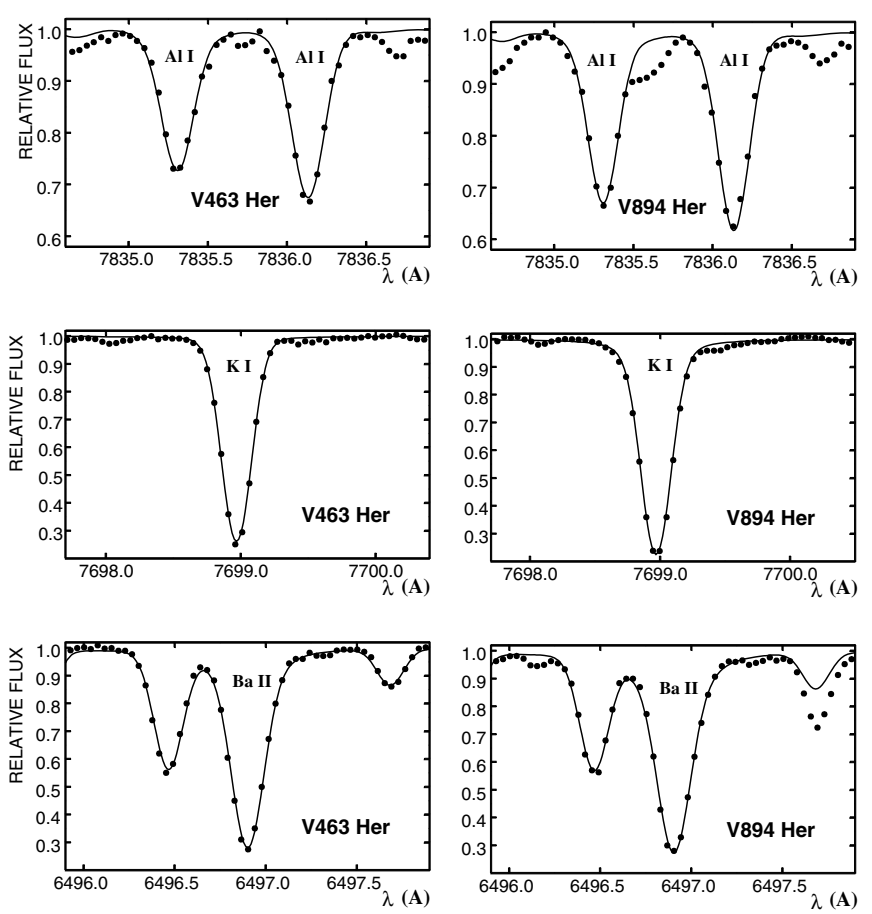

Fig. 4. Some examples of the NLTE line profile fitting for observed Al I, $\mathrm{K} \mathrm{I}$, and $\mathrm{Ba}$ II lines.

\section{Discussion and conclusion}

Our results presented in Tables 2 and 3 show that all investigated stars have elemental abundances that are not typical of the halo population. In Fig. 5 we compare the elemental abundances of the four stars from the present study with abundances in the stars studied by Andrievsky et al. (2007a - Paper I). As it was shown in that paper, all studied stars appeared to be halo Population II objects. From Fig. 5 it is evident that the difference between 
Table 2. Relative-to-solar elemental abundances in V463 Her and V894 Her.

\begin{tabular}{|c|c|c|c|c|c|c|c|c|c|c|}
\hline \multirow[b]{2}{*}{ Ion } & \multirow[b]{2}{*}[\mathrm{El}/\mathrm{H}]{$\mathrm{LTE}$} & \multirow{2}{*}{$\begin{array}{c}\text { V463 Her } \\
{[\mathrm{El} / \mathrm{H}] \text { NLTE }}\end{array}$} & \multirow[b]{2}{*}{$\sigma$} & \multirow[b]{2}{*}{ NL } & \multirow[b]{2}{*}[\mathrm{El}/\mathrm{Fe}]{} & \multicolumn{2}{|c|}{ V894 Her } & \multirow[b]{2}{*}{$\sigma$} & \multirow[b]{2}{*}{ NL } & \multirow[b]{2}{*}[\mathrm{El}/\mathrm{Fe}]{} \\
\hline & & & & & & {$[\mathrm{El} / \mathrm{H}] \mathrm{LTE}$} & {$[\mathrm{El} / \mathrm{H}] \mathrm{NLTE}$} & & & \\
\hline Li I & -0.89 & & - & 1 & -0.95 & -1.30 & & - & 1 & -1.27 \\
\hline $\mathrm{CI}_{\mathrm{I}}$ & +0.00 & -0.20 & 0.12 & 5 & -0.26 & -0.36 & -0.50 & 0.15 & 5 & -0.47 \\
\hline O I & +0.09 & +0.00 & 0.08 & 6 & -0.06 & +0.19 & +0.10 & 0.08 & 7 & +0.13 \\
\hline $\mathrm{NaI}$ & +0.32 & +0.15 & 0.08 & 4 & +0.09 & +0.29 & +0.15 & 0.08 & 4 & +0.18 \\
\hline Mg I & +0.04 & +0.06 & 0.08 & 6 & +0.00 & +0.10 & +0.11 & 0.08 & 5 & +0.14 \\
\hline $\mathrm{Al} \mathrm{I}$ & +0.13 & +0.00 & 0.08 & 3 & -0.06 & +0.25 & +0.15 & 0.08 & 3 & +0.18 \\
\hline Si I & +0.23 & & 0.22 & 45 & +0.17 & +0.03 & & 0.25 & 46 & +0.06 \\
\hline Si II & +0.03 & & 0.12 & 2 & -0.03 & +0.30 & & - & 1 & +0.33 \\
\hline S I & +0.23 & +0.00 & 0.07 & 3 & -0.06 & -0.01 & -0.20 & 0.07 & 3 & -0.17 \\
\hline $\mathrm{K}_{\mathrm{I}}$ & +0.31 & -0.01 & 0.07 & 2 & -0.07 & +0.61 & +0.39 & 0.07 & 2 & +0.42 \\
\hline Ca I & +0.10 & & 0.14 & 23 & +0.04 & +0.28 & & 0.20 & 20 & +0.31 \\
\hline Sc I & -0.24 & & 0.04 & 3 & -0.30 & +0.07 & & 0.22 & 9 & +0.10 \\
\hline $\mathrm{Sc}$ II & -0.20 & & 0.15 & 8 & -0.26 & -0.05 & & 0.20 & 17 & -0.02 \\
\hline Ti I & -0.02 & & 0.18 & 63 & -0.08 & +0.07 & & 0.16 & 49 & +0.10 \\
\hline Ti II & -0.00 & & 0.14 & 6 & -0.06 & +0.17 & & 0.06 & 6 & +0.20 \\
\hline$V_{\text {I }}$ & -0.06 & & 0.14 & 10 & -0.12 & +0.10 & & 0.21 & 25 & +0.13 \\
\hline Cr I & +0.20 & & 0.18 & 10 & +0.14 & -0.06 & & 0.18 & 26 & -0.03 \\
\hline Cr II & -0.07 & & 0.14 & 5 & -0.13 & -0.22 & & 0.12 & 8 & -0.19 \\
\hline Mn I & -0.11 & & 0.23 & 6 & -0.17 & -0.15 & & 0.13 & 5 & -0.12 \\
\hline $\mathrm{Fe} \mathrm{I}$ & +0.06 & & 0.21 & 165 & - & -0.03 & & 0.13 & 280 & - \\
\hline Fe II & +0.02 & & 0.07 & 9 & - & +0.03 & & 0.08 & 7 & - \\
\hline CoI & -0.02 & & 0.17 & 23 & -0.08 & +0.13 & & 0.22 & 16 & +0.16 \\
\hline Ni I & +0.19 & & 0.20 & 42 & +0.13 & +0.03 & & 0.20 & 44 & +0.06 \\
\hline $\mathrm{Cu} \mathrm{I}$ & +0.07 & & 0.18 & 3 & +0.01 & +0.30 & & - & 1 & +0.33 \\
\hline $\mathrm{Rb}$ I & -0.26 & & - & 1 & -0.32 & -0.03 & & - & 1 & +0.00 \\
\hline$Y_{I}$ & -0.01 & & 0.14 & 2 & -0.07 & +0.00 & & - & 1 & +0.03 \\
\hline Y II & +0.01 & & - & 1 & -0.05 & -0.24 & & 0.16 & 7 & -0.21 \\
\hline $\mathrm{ZrI}$ & -0.27 & & - & 1 & -0.33 & -0.29 & & - & - & -0.26 \\
\hline Zr II & -0.24 & & 0.22 & 2 & -0.30 & -0.43 & & 0.18 & 2 & -0.40 \\
\hline Mo I & +0.05 & & 0.12 & 2 & -0.01 & +0.11 & & 0.10 & 3 & +0.14 \\
\hline Ba II & +0.33 & +0.30 & 0.09 & 3 & +0.24 & +0.54 & +0.52 & 0.06 & 3 & +0.55 \\
\hline La II & -0.02 & & 0.04 & 3 & -0.08 & -0.34 & & 0.22 & 2 & -0.31 \\
\hline Ce II & -0.20 & & 0.12 & 11 & -0.26 & -0.14 & & 0.19 & 2 & -0.11 \\
\hline Pr II & +0.06 & & 0.12 & 3 & +0.00 & - & & - & - & - \\
\hline Nd II & -0.13 & & 0.10 & 11 & -0.19 & -0.29 & & 0.23 & 9 & -0.26 \\
\hline Eu II & -0.11 & & 0.18 & 6 & -0.17 & -0.27 & & 0.19 & 4 & -0.24 \\
\hline
\end{tabular}

Notes. NL - the number of lines used in our analysis.

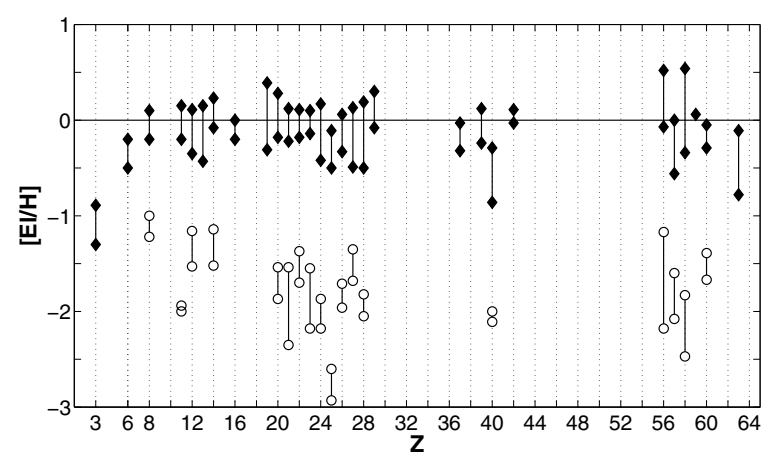

Fig. 5. Comparison of the abundance distributions in our programme stars and the halo-population SRd stars studied in Andrievsky et al. (2007a). Halo SRd stars (Andrievsky et al. 2007a) - circles; present programme stars - filled diamonds. For each chemical element the range comprising the abundances in all programme stars is indicated. $Z$ is the atomic number.

the chemical composition of the two groups is considerable. The stars of our present sample show all the signs of the thick/thin Galactic disc population objects. This is shown in Fig. 6, where we compared for each studied element (only common elements

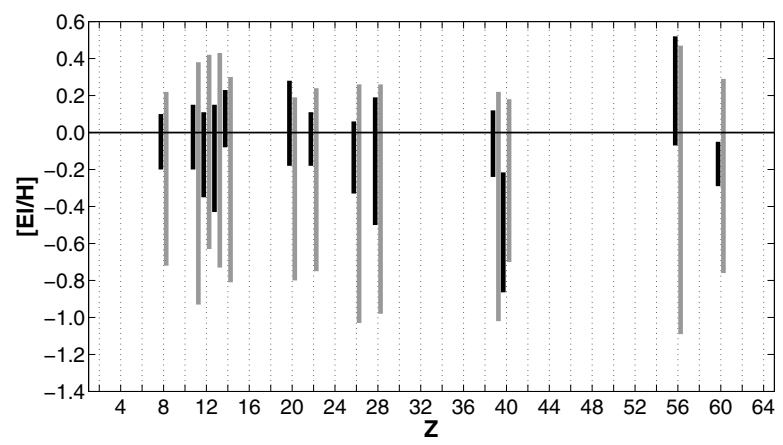

Fig. 6. Comparison of the abundance range for a given chemical element in our programme stars (black blocks) with that of the disc stars (grey blocks). The data for disc stars are from Edvardsson et al. (1993).

are showed) the range of abundance in our programme stars with that in the disc population stars investigated by Edvardsson et al. (1993). As one can see, the range of abundances of a given element in our program stars is within the range of abundances in the disc stars. 
Table 3. Relative-to-solar elemental abundances in CW CVn and MS Hya.

\begin{tabular}{|c|c|c|c|c|c|c|c|c|c|c|}
\hline \multirow[b]{2}{*}{ Ion } & \multirow[b]{2}{*}[\mathrm{El}/\mathrm{H}]{$\mathrm{LTE}$} & \multirow{2}{*}{$\begin{array}{c}\text { CW CVn } \\
{[\text { El/H] NLTE }}\end{array}$} & \multirow[b]{2}{*}{$\sigma$} & \multirow[b]{2}{*}{ NL } & \multicolumn{3}{|c|}{ MS Hya } & \multirow[b]{2}{*}{$\sigma$} & \multirow[b]{2}{*}{ NL } & \multirow[b]{2}{*}[\mathrm{El}/\mathrm{Fe}]{} \\
\hline & & & & & {$[\mathrm{El} / \mathrm{Fe}]$} & {$[\mathrm{El} / \mathrm{H}] \mathrm{LTE}$} & {$[\mathrm{El} / \mathrm{H}] \mathrm{NLTE}$} & & & \\
\hline OI & -0.20 & -0.20 & 0.15 & 1 & -0.06 & +0.00 & +0.00 & 0.15 & 3 & +0.33 \\
\hline $\mathrm{Na}$ I & +0.01 & -0.20 & 0.12 & 4 & -0.06 & +0.32 & +0.05 & 0.10 & 3 & +0.38 \\
\hline Mg I & -0.05 & -0.09 & 0.15 & 3 & +0.05 & -0.26 & -0.35 & 0.15 & 4 & -0.02 \\
\hline Al I & -0.21 & -0.43 & 0.15 & 2 & -0.29 & -0.02 & -0.30 & 0.10 & 3 & +0.03 \\
\hline Si I & -0.08 & & 0.18 & 4 & +0.06 & +0.16 & & 0.10 & 8 & +0.49 \\
\hline K I & -0.11 & -0.31 & 0.12 & 2 & -0.17 & +0.13 & -0.01 & 0.10 & 2 & +0.32 \\
\hline $\mathrm{Ca} \mathrm{I}$ & -0.18 & & 0.16 & 7 & -0.04 & +0.02 & & 0.14 & 11 & +0.35 \\
\hline $\mathrm{ScI}$ & - & & - & - & - & +0.19 & & 0.11 & 2 & +0.52 \\
\hline Sc II & +0.12 & & 0.05 & 2 & +0.26 & +0.02 & & 0.10 & 3 & +0.35 \\
\hline Ti I & -0.17 & & 0.14 & 8 & -0.03 & -0.17 & & 0.09 & 20 & +0.16 \\
\hline Ti II & +0.08 & & 0.02 & 2 & +0.22 & -0.29 & & 0.19 & 3 & +0.04 \\
\hline V I & -0.14 & & 0.15 & 6 & +0.00 & -0.04 & & 0.20 & 10 & +0.29 \\
\hline CrI & - & & - & - & - & -0.42 & & 0.04 & 3 & -0.09 \\
\hline Mn I & -0.50 & & 0.07 & 3 & -0.36 & -0.38 & & 0.17 & 2 & -0.05 \\
\hline $\mathrm{Fe} I$ & -0.14 & & 0.22 & 79 & - & -0.33 & & 0.22 & 140 & - \\
\hline Fe II & - & & - & - & - & -0.30 & & 0.09 & 3 & - \\
\hline Co I & -0.18 & & 0.11 & 6 & -0.04 & -0.49 & & 0.21 & 8 & -0.16 \\
\hline Ni I & -0.50 & & 0.20 & 10 & -0.36 & +0.17 & & 0.19 & 18 & +0.50 \\
\hline $\mathrm{Cu} \mathrm{I}$ & - & & - & - & - & -0.08 & & 0.25 & 2 & +0.25 \\
\hline $\mathrm{Rb}$ I & -0.02 & & - & 1 & +0.12 & -0.32 & & 0.20 & 2 & +0.01 \\
\hline Y II & +0.12 & & 0.2 & 2 & +0.26 & -0.24 & & - & 1 & +0.09 \\
\hline ZrI & -0.86 & & - & 1 & -0.72 & -0.31 & & 0.13 & 4 & +0.02 \\
\hline Mo I & - & & - & - & - & -0.03 & & - & 1 & +0.30 \\
\hline Ba II & -0.10 & -0.07 & 0.12 & 3 & +0.07 & +0.29 & +0.32 & 0.10 & 3 & +0.65 \\
\hline La II & -0.56 & & 0.07 & 2 & -0.42 & +0.00 & & - & 1 & +0.33 \\
\hline Ce II & +0.54 & & 0.11 & 3 & +0.68 & -0.34 & & 0.19 & 3 & -0.01 \\
\hline Nd II & -0.25 & & 0.03 & 2 & -0.11 & -0.05 & & 0.18 & 16 & +0.28 \\
\hline Eu II & -0.78 & & - & 1 & -0.64 & -0.29 & & - & 1 & +0.04 \\
\hline
\end{tabular}

Edvardsson et al. did not derive the abundances of the elements such as lithium and carbon, for example. The reduced carbon (and lithium) abundance in V463 Her and V894 Her (Table 2) suggests that these giants might have already experienced the dredge-up episode, which brings material that was processed in the incomplete $\mathrm{CNO}$ cycle to the surface.

Summarizing, after spectroscopically investigating four reliably classified SRd stars and taking into account the results of previous quantitative analyses of other stars of this class, we can conclude that we obtained convincing evidence that the SRd class is chemically inhomogeneous. It comprises the stars of both halo and disc population objects, which can manifest a similar photometric semiregular variability.

Acknowledgements. S.M.A. gratefully acknowledges the CNRS for financial support and the administration of the Paris-Meudon Observatoire for its hospitality during his stay in Meudon, where part of this work was performed. We acknowledge the CFHT Support Staff for their help during the observations. The authors thank the anonymous referee for offering important suggestions.

\section{References}

Andrievsky, S. M., Makarenko, E. N., \& Fenina, Z. N. 1985, PrKFi, 20, 60 Andrievsky, S. M., Kovtyukh, V. V., Korotin, S. A., Spite, M., \& Spite, F. 2001, A\&A, 367, 605

Andrievsky, S. M., Korotin, S. A., \& Martin, P. 2007a, A\&A, 464, 709 (Paper I) Andrievsky, S. M., Spite, M., Korotin, S. A., et al. 2007b, A\&A, 464, 1081 Andrievsky, S. M., Spite, M., Korotin, S. A., et al. 2008, A\&A, 481, 481 Andrievsky, S. M., Spite, M., Korotin, S. A., et al. 2009, A\&A, 494, 1083 Andrievsky, S. M., Spite, M., Korotin, S. A., et al. 2010, A\&A, 509, A88 Carlsson, M. 1986, Uppsala Obs. Rep., 33

Crimi, G., \& Mantegazza, L. 1984, Ap\&SS, 100, 255

Edvardsson, B., Andersen, J., Gustafsson, B., et al. 1993, A\&A, 275, 101 Galazutdinov, G. A. 1992, Preprint SAO RAS, n92

Giridhar, S., Lambert, D., \& Gonzalez, G. 1998, PASP, 110, 671 Giridhar, S., Lambert, D., \& Gonzalez, G. 1999, PASP, 111, 1269 Giridhar, S., Lambert, D., \& Gonzalez, G. 2000, PASP, 112, 1559 Korotin, S. A., \& Mishenina, T. V. 1999, ARep, 43, 533

Korotin, S. A., Andrievsky, S. M., \& Luck, R. E. 1999a, A\&A, 351, 168

Korotin, S. A., Andrievsky, S. M., \& Kostynchuk, L. Yu. 1999b, Ap\&SS, 260, 531

Kovtyukh, V. V., \& Andrievsky, S. M. 1999, A\&A, 351, 597

Kovtyukh, V. V., Mishenina, T. V., Gorbaneva, T. I., et al. 2006, ARep, 50, 134 Kurucz, R. L. 1992, RMxAA, 23,45

Tsymbal, V. V. 1996, Model Atmospheres and Spectrum Synthesis, ed. S. J. Adelman, F. Kupka, \& W. W. Weiss (San Francisco), ASP Conf. Ser., 108 Walhgren, G. M. 2003, ASPC, 298, 267 